\title{
Drei Jahre, fünf Jahre oder ein Leben lang?
}

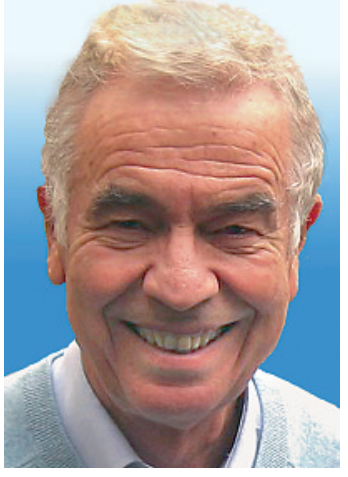

Hans Stalder
Die Weiterbildung zur Erlangung des Facharzttitels in Allgemein-/Hausarztmedizin ist in den verschiedenen Ländern von unterschiedlicher Dauer: In einigen Staaten sind nur zwei Jahre erforderlich, mehrere EU-Länder sowie die USA haben drei Jahre festgelegt, andere wie Deutschland und Dänemark fünf. In der Schweiz kämpft die Schweizerische Gesellschaft für Allgemeinmedizin (SGAM) für den Erhalt der ebenfalls fünfjährigen Weiterbildung. Dazu ist man sogar bereit, den Facharzttitel in Allgemeinmedizin gegen den der Inneren Medizin einzutauschen, was der Gesellschaft ihre Mitgliedschaft in der Wonca kosten könnte. Grund ist der freie Personenverkehr zwischen der EU und der Schweiz. Denn die Mindestweiterbildungsdauer der Hausarztmedizin in der EU beträgt nur drei Jahre, und das erachtet die SGAM als unzureichend: Undenkbar, sich mit Kollegen auf eine Stufe zu stellen, die ihren Titel im Rabatt von nur drei Jahren erhalten haben!

Sind die schweizerischen und deutschen Allgemeinmediziner aber wirklich soviel besser als beispielsweise die englischen oder niederländischen? Das ist zumindest fraglich! Vergleichende Untersuchungen gibt es nur in Bezug auf die Patientenzufriedenheit, und hier liegen die Schweizer Ärzte in der Tat vorne. Allerdings scheint es, dass die Patientenzufriedenheit eher von der Erreichbarkeit des Arztes und der freien Arztwahl als von der Qualität der Leistung abhängig ist.

Wenn wir einräumen, dass die kürzere Weiterbildungszeit, zumindest in einigen Ländern, ebenso gute Ärzte hervorbringt wie bei uns, stellt sich die Frage, wie diese Länder es schaffen, hochqualifizierte Allgemeinmediziner in nur drei Jahren auszubilden. Es gibt mehrere mögliche Antworten: Schon im Studium könnten auf dem Gebiet der Hausarztmedizin höhere Ansprüche gestellt werden. Damit wären die Kandidaten auf die Weiterbildung in Allgemeinmedizin besser vorbereitet. Dies gilt für die Niederlande und Grossbritannien, wo die Hausarztmedizin an den medizinischen Fakultäten sehr einflussreich ist und dadurch ihre Ausbildung stark beeinflussen kann.

Die wichtigste Voraussetzung für die Weiterbildung hervorragender Hausärzte innert nur dreier Jahre ist allerdings, dass klare Ausbildungsziele festgelegt werden, eine diesen Zielen entsprechende Bewertung stattfindet und dass vor allem angemessene pädagogische und finanzielle Mit- tel zur Umsetzung der Ziele bereitgestellt werden. So dauert in den Niederlanden und in Grossbritannien die Weiterbildung in der Praxis drei- bis viermal länger als die im Spital, und die Hausärzte werden gut auf ihre Aufgabe als Lehrärzte vorbereitet. Die Fakultäten sind in den strukturierten Weiterbildungszyklus eingebunden; dieser umfasst in den Niederlanden Lehrveranstaltungen und Seminare.

Dazu kommt, dass, während früher die Weiterbildung nach Erlangung des Facharzttitels abgeschlossen war, heute Lernen ein Prozess ist, der das ganze Berufsleben währt. Auf die ärztliche Weiterbildung muss automatisch die Fortbildung folgen. Und auch diese muss strukturiert sein, bewertet werden (nicht unbedingt in Form obligatorischer Prüfungen) und vor allem anerkannt sein, was bei uns nur selten der Fall ist.

Zweifellos muss die Weiterbildung eine gewisse Zeit dauern. Jedoch ist nicht die Zeit an sich entscheidend - denn in jedem Fall ist Lernen ein lebenslanger Prozess - sondern die Qualität: Wichtig ist, dass die Zielsetzungen klar definiert, die entsprechenden Mittel eingesetzt und eine fachgerechte Bewertung sichergestellt werden. Die Ausbildung in Allgemeinmedizin muss am ersten Tag des Studiums beginnen und darf erst mit der Beendigung der Berufslaufbahn abschliessen - mit einer «ärztlichen Weiterbildung». In der Schweiz nicht in drei Jahren realisierbar? Wenn es in anderen Ländern möglich ist, warum dann nicht auch bei uns? Warum sollten wir es nicht zumindest einmal gedanklich durchspielen und uns zum Beispiel die Frage stellen, ob das Wahljahr unbedingt notwendig ist: Erfüllt es wirklich die Weiterbildungsziele, nämlich die Ausbildung eines guten Allgemeinmediziners? Mit seiner Einsparung hätte man bereits ein Jahr gewonnen ...

Solche Überlegungen zu machen, wären eine interessante Aufgabe für die neuen universitären Institute für Hausarztmedizin: Unter welchen Bedingungen wäre es möglich, in der Schweiz hervorragende Allgemeinmediziner in nur drei Jahren auszubilden? Wie wäre dies durchführbar? Wo sind die Hindernisse? Zumindest würde dies den Hausärzten erlauben, nicht «Spezialisten» zu werden, sondern ihren schönen, europaweit vertretenen Titel Allgemeinmedizin zu behalten.

Hans Stalder* von Internisten und Pädiatern ebenfalls nur drei Jahre.

S: Selbstverständlich gelten dieselben Argumente auch für die Ausbildung in den anderen Disziplinen der Hausarztmedizin. Zum Beispiel 